\title{
CORRIGENDUM
}

\section{Search for Electromagnetic Counterparts to LIGO-Virgo Candidates: Expanded Very Large Array Observations - CORRIGENDUM}

Joseph Lazio, Katie Keating, F. A. Jenet and N. E. Kassim

doi:10.1017/S1743921312000245, Published by Cambridge University Press, 20 April 2012 .

In the article by Joseph Lazio, Katie Keating, F. A. Jenet and N. E. Kassim published in Proceedings IAU Symposium No. 285 there was an error in the listing of authors. The correct authorship is:

Joseph Lazio, Katie Keating, F. A. Jenet, and N. E. Kassim, the LIGO Scientific Collaboration and the Virgo Collaboration

\section{Reference}

Lazio, J., Keating, K., Jenet, F. A. and Kassim, N. E. 2011, IAU Symp. No. 285, pp. 67-70 doi:10.1017/S1743921312000245 